\title{
Endoscopic submucosal dissection as a treatment for gastric subepithelial tumors that originate from the muscularis propria layer: a preliminary analysis of appropriate indications
}

\author{
Seung Yeon Chun · Kyoung Oh Kim • Dong Seon Park • In Joung Lee • \\ Ji Won Park • Sung-Hoon Moon • Il Hyun Baek • Jong Hyeok Kim • \\ Choong Kee Park $\cdot$ Mi Jung Kwon
}

Received: 27 September 2012/ Accepted: 15 February 2013/Published online: 22 March 2013

(C) The Author(s) 2013. This article is published with open access at Springerlink.com

\begin{abstract}
Background Endoscopic submucosal dissection (ESD) is a well-established method for the treatment of gastrointestinal epithelial tumors. However, the treatment of gastric subepithelial tumors (SETs) that originate from the muscularis propria layer still depends primarily on surgical techniques. We evaluated the appropriate indications for ESD in the treatment of SETs that originate from the muscularis propria layer.

Methods Thirty-five patients with gastric SETs that originate from the muscularis propria layer who underwent ESD were enrolled, and the charts were retrospectively reviewed to investigate the parameters predictive complete resection and complications.

Results The mean age of the patients was $54.15 \pm$ 9.3 years, and the male/female ratio was $2: 3$. Twenty-eight of the 35 SETs $(85.7 \%)$ were movable, and $15(45.7 \%)$ had a positive rolling sign. The most frequent location of the SETs was high body $(n=14)$. The most common pathological
\end{abstract}

S. Y. Chun · K. O. Kim $(\bowtie) \cdot$ D. S. Park ·

I. J. Lee · J. W. Park - S.-H. Moon - I. H. Baek ·

J. H. Kim - C. K. Park

Division of Gastroenterology and Hepatology, Department of Internal Medicine, Hallym University Sacred Heart Hospital, Hallym University, College of Medicine, 896, Pyeongchondong, Dongan-gu, Anyang, Gyeonggi-do 431-070, Korea e-mail: kkoimge@naver.com

K. O. Kim

Department of Internal Medicine, Gachon University Gil Medical Center, Incheon, Korea

M. J. Kwon

Department of Pathology, Hallym University Sacred Heart Hospital, Hallym University, College of Medicine, Anyang, Korea diagnoses were leiomyoma (60 \%) and gastrointestinal stromal tumor $(28.6 \%)$. The complete resection rate was $74.3 \%$. A positive rolling sign $(p=0.022)$ and small tumor size $(\leq 20 \mathrm{~mm} ; p=0.038)$ were significantly associated with complete resection. Two patients $(6.1 \%)$ developed perforations that required surgical treatment; their SMTs were neurogenic tumors with fixed lesion. Tumor mobility was significantly associated with perforation $(p=0.017)$.

Conclusions The ESD method appears to be relatively safe for use in the complete resection of SETs that originate from the muscularis propria layer. Small tumor size $(\leq 20 \mathrm{~mm})$ and a positive rolling sign are appropriate indications for ESD.

Keywords Subepithelial tumor - Muscularis propria . Endoscopic submucosal dissection

Gastric subepithelial tumors (SETs) are common lesions in the upper gastrointestinal tract and often are found incidentally during endoscopic examination [1]. SETs often are considered to be relatively benign; however, they have malignant potential, particularly if they originate from the muscularis propria layer [2]. Gastrointestinal stromal tumor (GIST), the most common neoplasm that originates from the muscularis propria layer of the gastrointestinal tract, is diagnosed as malignant in 10-30\% of cases. The nature of GISTs cannot be determined based solely on endoscopic imaging [3, 4]. Endoscopic ultrasound (EUS) is the most accurate method for differentiating gastrointestinal SETs; however, in most cases, it is not sufficient for making a definite diagnosis of hypoechoic lesions in the third or fourth echo layer $[5,6]$. An accurate pathological confirmation therefore is necessary for differentiating between benign and malignant SETs. 
Many tissue sampling techniques have been developed for the definitive diagnosis of SETs, including bite-on-bite biopsy, EUS-guided fine needle aspiration (EUS-FNA), and EUS-guided core needle biopsy [2, 4]. However, these techniques are not always sufficient for diagnosis, and normal pathological reports from EUS-FNA or EUSguided core needle biopsy cannot rule out the possibility of malignancy. More aggressive techniques for tissue diagnosis, such as tumor biopsy following incision or unroofing (partial removal of the overlying mucosa) and endoscopic submucosal-mucosal resection (ESMR), have a better diagnostic yield; however, the available data on the effectiveness of these techniques are limited [7-9].

Endoscopic submucosal dissection (ESD) has been developed in recent years as a technique for the treatment of early gastric cancer [10]. The ESD technique allows precise en bloc resection regardless of the size and shape of the lesion; however, evidence regarding the efficacy and safety of ESD in the resection of SETs is limited. An initial study suggested that complete en bloc resection using ESD is indicated only for SETs that originate from the muscularis mucosa or submucosal layer [8]. ESD for the resection of SETs that arise from the muscularis propria layer has been recently introduced in several feasibility studies and has shown a complete resection rate of approximately 64-75 \% [11-13]. However, ESD appears to be associated with technical difficulty and a higher incidence of complications, such as perforation and bleeding, and the treatment of SETs that originate from the muscularis propria layer still depends primarily on surgical techniques.

In this study, we evaluated the appropriate indications for ESD in SETs that originate from the muscularis propria layer. We retrospectively reviewed data from patients who underwent ESD for gastric SETs to determine the predictors of complete resection and the possible complications of ESD.

\section{Materials and methods}

Patients and study design

Between March 2009 and May 2012, 35 ESD procedures were performed in consecutive patients at the Hallym University Sacred Heart Hospital, Anyang, Korea, who had gastric SETs that originated from the muscularis propria layer. Patients were considered eligible for ESD if the tumor originated from the muscularis propria layer and did not show extraluminal growth as confirmed by EUS. The data were collected retrospectively, and the charts were reviewed for the following information: clinical characteristics, endoscopic and EUS findings, and clinical outcomes.
All of the patients included in the study provided written, informed consent to undergo ESD following detailed verbal and written explanations of the ESD procedure and other possible treatment options. This study was approved by the institutional review board of the hospital.

\section{Standard endoscopy and EUS}

All of the patients underwent a routine upper GI endoscopy (GIF-Q260; Olympus Optical Co., Tokyo, Japan). The assessment of each tumor included the location, size, appearance, extent, mobility, and consistency of the lesion. The location of the lesion was classified based on six sections: cardia, fundus, high body, midbody, lower body, and antrum. When the tumor was freely movable more than half of maximal tumor diameter upon palpation with biopsy forceps, the lesion was considered to have a positive rolling sign.

EUS was performed with a radial-scanning echo endoscope (Olympus GF-UM2000, $6 \mathrm{MHz}$ and $12 \mathrm{MHz}$; Olympus Optical Co.) to assess the layer of origin. Other features that were identified by EUS included the growth pattern (intragastric vs. extragastric), the demarcation of margin, and the degree of the connection area (narrow vs. wide) with the fourth layer of the tumor. A narrow versus wide muscular connection was defined as a connection area with the fourth layer that was $\leq 50$ versus $>50 \%$ of the maximal diameter of the tumor base, respectively (Fig. 1) [11].

\section{ESD procedures}

Before the ESD procedure, each patient was sedated using an intravenous propofol infusion to produce a deep sedation level and his/her heart rate, blood pressure, and oxygen saturation were closely monitored. The ESD procedure was performed using a video endoscope (GIF-Q260J; Olympus Optical Co.) with a hood (length, $4 \mathrm{~mm}$ ) attached at the endoscope tip. After the target lesion was identified, marking dots were placed circumferentially at its margin using an argon plasma coagulation (APC) probe under an electrosurgical coagulation current (forced coagulation, $40 \mathrm{~W}$ ). A mucosal incision was made with a needle-knife (KD-10Q-1L; Olympus Optical Co.) along the line of dots following the injection of a mixture of glycerol (10\%), epinephrine (dilution 1:100,000), and a small amount of indigo carmine. An insulated-tip knife (KD-611; Olympus Optical Co.) was inserted into the initial incision, and an electrosurgical current was applied using an electrosurgical generator (Erbe VIO 300D; Tübingen, Germany) in EndoCut mode (effect 2) to make an incision around the lesion. 




Fig. 1 The degree of muscular connection with a subepithelial tumor as shown by endoscopic ultrasound. A Narrow muscular connection with the tumor: the diameter of the connection with the fourth layer of the tumor is $<50 \%$ of the maximal diameter of the tumor base (black arrow

After the circumferential incision of the overlying mucosa, the tumor was exposed below the submucosal layer. The insulated-tip knife (coagulation mode, effect 5 , $60 \mathrm{~W}$ ) was used in combination with additional saline and indigo carmine injections to separate carefully the tumor from the muscularis propria layer. The procedure time, defined as the required time from the marking to the resection of the lesion, was recorded for each procedure. The resection wound was observed carefully to evaluate whether any residual tumor was present.

Pathological examination and follow-up assessment

The tissue specimens were fixed in formalin solution. The histological evaluation included the identification of cell type, overall cellularity, nuclear atypia, immunohistochemical findings, and the mitotic index. Immunohistochemical analyses of c-kit (CD 117), CD 34, smooth muscle actin (SMA), and S-100 markers were performed to identify the tumor subtype. The resection margin was examined microscopically, and its status was determined on the basis of the general criteria of cancer involvement in resection margin [14]. The completeness of resection was classified according to the extension of tumor cells into the resection margin: (1) complete (R0) resection, in which the lateral and vertical resection margins were free of tumor; (2) microscopic incomplete (R1) resection, in which the tumor extended into the lateral or vertical resection margin; and (3) macroscopic incomplete (R2) resection, in which the tumor could not be completely resected according to its endoscopic aspects (Fig. 2).

Each patient who underwent ESD was followed up by standard endoscopy within the 2-month period following

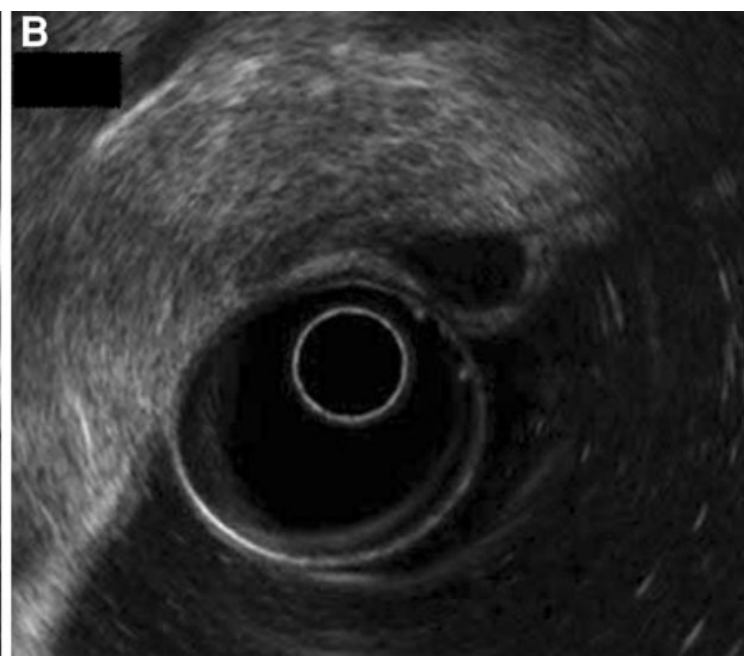

hyperechoic demarcation line between the tumor and the muscularis propria layer). B Wide muscular connection with the tumor: the diameter of the connection with the fourth layer is $>50 \%$ of the maximal diameter of the tumor base

the procedure to confirm the healing of the artificial ulcer and to rule out local recurrence. The patients who had GISTs underwent surveillance examinations every 6 months, and surveillance studies included both endoscopy and computed tomography of abdomen and pelvis.

\section{Outcome measurements}

The major outcome measurements were complete resection rate, procedure-related complication rate, and diagnostic parameters predictive of complete resection. The complete resection rate was defined as the proportion of tumors that were removed en bloc by R0 resection. Procedure-related complications included perforation and bleeding. Perforation was diagnosed when mesenteric fat or the intra-abdominal space was directly observed during the procedure (frank perforation) or when free air was observed on a plain chest X-ray following the procedure. If an endoscopically visible perforation was observed during the procedure, it was closed with hemoclips and additional surgical management was recommended. Major bleeding was defined as continued bleeding that required the administration of $\geq 2 \mathrm{U}$ of packed RBCs (PRBCs) or uncontrolled bleeding that persisted despite endoscopic intervention with at least two hemostatic modalities.

\section{Statistical analyses}

The $\chi^{2}$ test or Fisher's exact test was used for comparisons of categorical data, and the $t$ test was used for the analysis of continuous data. A $p$ value of $<0.05$ was considered to be statistically significant. All of the data were analyzed 

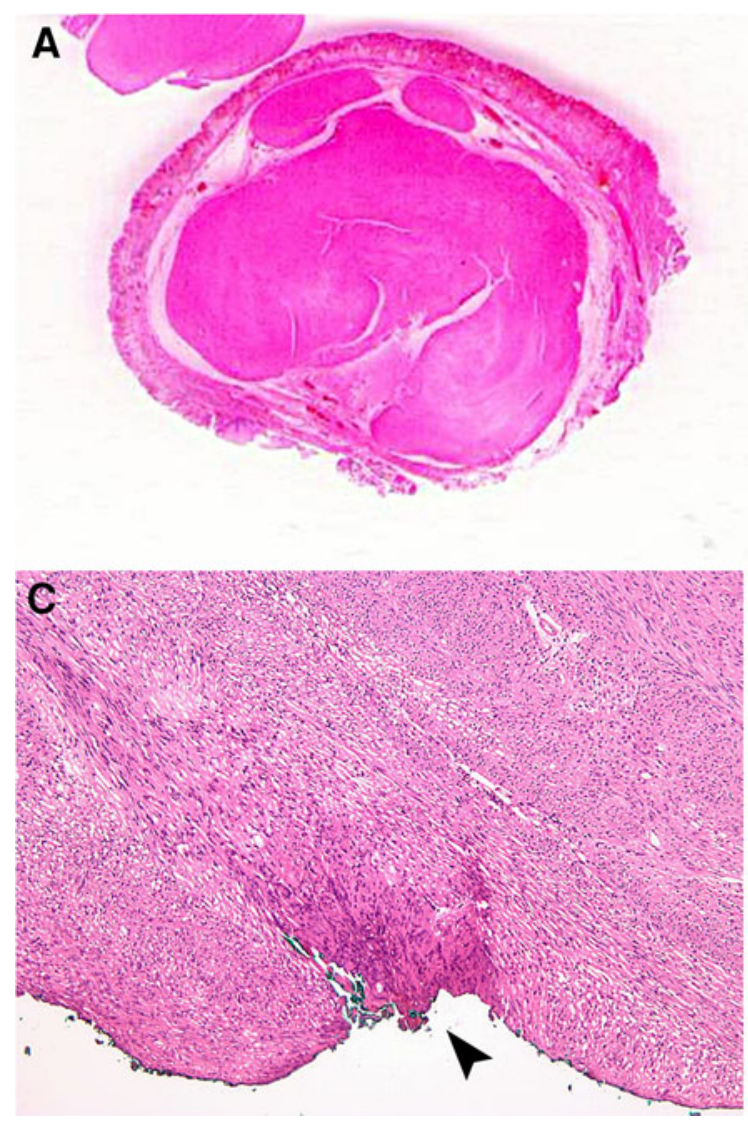

Fig. 2 A Magnified scan of an H\&E slide showing a well-demarcated submucosal tumor with clear circumferential resection margins following endoscopic submucosal dissection. B The deep resection

using the SPSS software program (version 16.0 for Windows; SPSS Korea, Seoul, Korea).

\section{Results}

Characteristics of the patients and the tumors

A total of 35 adult patients with gastric SETs underwent ESD at Hallym University Sacred Heart Hospital. The characteristics of these patients are summarized in Table 1. The mean age of the patients was $54.15 \pm 9.3$ years (range 31-71 years), and the male/female ratio was 2:3. The mean follow-up duration of the study subjects was $6.13 \pm 9.02$ months (range 1-44.3 months).

Standard upper GI endoscopy revealed that 30 of the 35 SETs $(85.7 \%)$ were movable by biopsy forceps and that 15 SETs $(45.7 \%)$ of these mobile tumors had a positive rolling sign. The most frequent location of the SETs was high body $(n=14,40 \%)$, followed by cardia $(n=10$, $28.6 \%)$, lower body $(n=4,11.4 \%)$, mid body $(n=3$, $8.6 \%)$, antrum $(n=3,8.6 \%)$, and fundus $(n=1,2.9 \%)$.

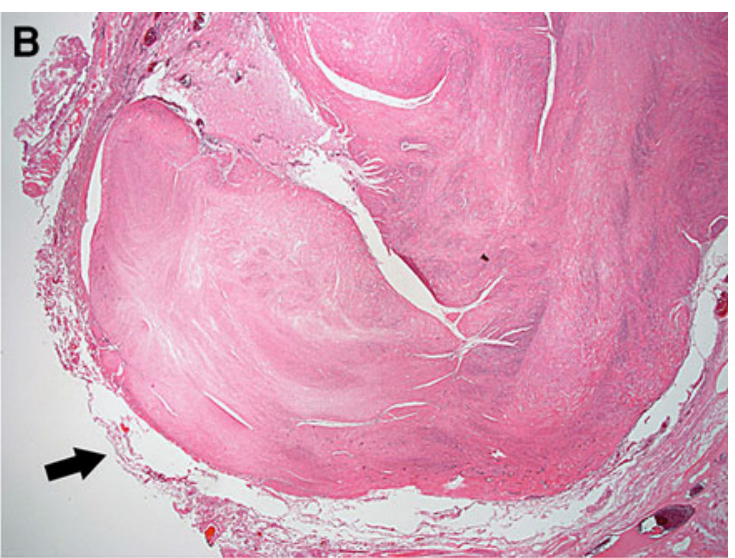

margin (arrow) is negative (R0) $(\times 40)$. C The resection margin, indicated by green color and an arrowhead, is involved with a gastrointestinal stromal tumor $(\mathrm{R} 1)(\times 200)$

EUS revealed a narrow connection with the fourth layer in 12 tumors $(34.3 \%)$ and a well-demarcated margin in 34 tumors $(97.1 \%)$.

Twenty-one patients $(60 \%)$ had leiomyomas, and ten patients $(28.6 \%)$ had GISTs. Other histological diagnoses included neurogenic tumor $(n=2)$, gastritis cystica profunda $(n=1)$, and inflammatory fibroid polyp $(n=1)$. The mean maximal diameter of the tumors was $17.99 \pm 7.86 \mathrm{~mm}$ (range $8-40 \mathrm{~mm}$ ). The HPF mitotic counts of most resected tumors were low ( $<5$ mitotic figures/50 HPF), suggesting a low risk of malignancy. Two SETs were found to have a greater than moderate risk of malignancy. One patient had a GIST of moderate risk (mitotic figures, 9/50 HPF; size, $2.2 \mathrm{~cm}$ ) with an R1 resection, but she refused additional surgical management. The other patient had a GIST with high risk (mitotic figures, 15/50 HPF; size, $2.2 \mathrm{~cm}$ ), and an R1 resection was performed. This patient underwent a gastric wedge resection and adjuvant chemotherapy with imatinib mesylate (Gleevec; Novartis Pharmaceuticals, Basel, Switzerland) for 1 year. No recurrence has been detected to date during follow-up (28 months). 
Table 1 Characteristics of the patients and the tumors $(n=35)$

\begin{tabular}{ll}
\hline Sex (female/male) & $14 / 21$ \\
Age (year; mean \pm SD) & $54.15 \pm 9.3$ \\
Follow-up duration (month; mean \pm SD) & $6.13 \pm 9.02$ \\
Endoscopic findings & \\
Rolling sign, $n(\%)$ & \\
Positive & $16(45.7 \%)$ \\
$\quad$ Negative & $19(54.3 \%)$ \\
Mobility, $n(\%)$ & \\
Fixed & $5(14.3 \%)$ \\
Mobile & $30(85.7 \%)$ \\
Location, $n(\%)$ & \\
Fundus & $1(2.9 \%)$ \\
Cardia & $10(28.6 \%)$ \\
High body & $14(40 \%)$ \\
Mid body & $3(8.6 \%)$ \\
Lower body & $4(11.4 \%)$ \\
Antrum & $3(8.6 \%)$ \\
Endoscopic ultrasound findings & \\
Connection with the fourth layer on EUS, $n(\%)$ & \\
Narrow & $12(34.3 \%)$ \\
Wide & $23(65.7 \%)$ \\
Margin of tumor & \\
Well-demarcated & \\
Not well-demarcated & $17.99 \pm 7.86$ \\
Pathological diagnosis & \\
Leiomyoma & \\
GIST & $21(60 \%)$ \\
Neurogenic tumor & \\
Other & \\
Tumor diameter (mm; mean \pm SD) & \\
\hline
\end{tabular}

Clinical outcomes

\section{Resection rate}

A successful complete resection (R0) by ESD was achieved in 26 of 35 tumors (success rate, $74.3 \%$ ). We analyzed the rate of complete resection according to tumor size, routine endoscopic and EUS features, and histological diagnosis (Table 2). The complete resection rate was significantly different in cases of a tumor size $\leq 20 \mathrm{~mm}$ than in cases of a tumor size $>20 \mathrm{~mm}$ (87 vs. $50 \%$, respectively; $p=0.038$ ). A positive rolling sign was significantly associated with more frequent complete resection (93.8 vs. $57.9 \%, p=0.022)$. The other parameters determined by EUS (demarcation of the margin and the degree of connection with the fourth echo layer) did not show a correlation with the complete resection rate.

\section{Complications}

The mean ESD procedure time was $32.29 \pm 20.55 \mathrm{~min}$ (range 7-84 min). No immediate postprocedure complications were observed. Two patients $(6.1 \%)$ developed perforations that required surgical treatment; no patient developed major bleeding (Table 3). In one of the cases of perforation, the tumor was tightly adherent to the muscularis propria layer, and an endoscopically visible perforation was observed during the procedure. An emergent operation was performed, and the final pathological diagnosis of the tumor was neurilemoma. In the other case of perforation, a visible perforation was observed after removal of the tumor. The patient underwent a wedge resection, and the pathological diagnosis of the tumor was schwannoma with a clear resection margin.

The predictive parameters of perforation are shown in Table 4. A positive rolling sign, small tumor size $(<20 \mathrm{~mm})$, and wide connection with the fourth echo layer on EUS were not significantly associated with perforation. Both cases of perforation involved fixed lesions, and tumor mobility was significantly associated with perforation $(p=0.017)$.

\section{Discussion}

The clinical application of ESD, including its application in the treatment of SETs that originate from the muscularis propria layer, has increased in recent years [11-13]. However, this technique is still in an experimental phase, and no studies have addressed its safety, long-term outcomes, or appropriate indications. Most studies to date on the endoscopic resection of SETs have been conducted on a small scale and have been concerned only with technical feasibility. However, a few recent studies have investigated the factors that are related to complete resection and to complications.

GISTs are the most common type of SET and are the primary target of treatment. GISTs are potentially malignant and have a high postoperative recurrence rate. It therefore is preferable that the purpose of treatment should be microscopic complete resection (R0 resection) with a microscopically negative margin rather than macroscopic complete resection. Because macroscopic complete resection has been considered to be equivalent to complete resection in most previous reports on the endoscopic resection of SET, the assessment of the usefulness of endoscopic resection has been limited.

Białek et al. [11] recently analyzed factors related to the rate of endoscopic complete resection (R0 resection) and to complications following ESD for SET. These authors reported that the area connected to the muscularis propria 
Table 2 Predictive parameters for complete resection in patients who underwent endoscopic submucosal dissection for gastric subepithelial tumors

\begin{tabular}{|c|c|c|c|}
\hline \multirow[t]{2}{*}{ Parameters } & \multicolumn{2}{|c|}{ Complete resection } & \multirow[t]{2}{*}{$p$ value } \\
\hline & Yes $(n=26)$ & No $(n=9)$ & \\
\hline Rolling sign, $n(\%)$ & & & 0.022 \\
\hline Yes & $15(93.8 \%)$ & $1(6.3 \%)$ & \\
\hline No & $11(57.9 \%)$ & $8(42.1 \%)$ & \\
\hline Mobility, $n(\%)$ & & & 0.095 \\
\hline Fixed lesion & $2(40 \%)$ & $3(60 \%)$ & \\
\hline Mobile lesion & $24(80 \%)$ & $6(20 \%)$ & \\
\hline Margin of tumor, $n(\%)$ & & & 0.257 \\
\hline Well-demarcated margin & $26(76.5 \%)$ & $8(23.5 \%)$ & \\
\hline No well-demarcated margin & $0(0 \%)$ & $1(100 \%)$ & \\
\hline Connection with the fourth layer on EUS, $n(\%)$ & & & 0.121 \\
\hline Narrow connection & $11(91.7 \%)$ & $1(8.3 \%)$ & \\
\hline Wide connection & $15(65.2 \%)$ & $8(34.8 \%)$ & \\
\hline Tumor size, $n(\%)$ & & & $\mathbf{0 . 0 3 8}$ \\
\hline$\leq 20 \mathrm{~mm}$ & $20(87 \%)$ & $3(13 \%)$ & \\
\hline$>20 \mathrm{~mm}$ & $6(50 \%)$ & $6(50 \%)$ & \\
\hline Histological diagnosis, $n(\%)$ & & & 0.291 \\
\hline Leiomyoma & $18(85.7 \%)$ & $3(14.3 \%)$ & \\
\hline GIST & $6(60 \%)$ & $4(40 \%)$ & \\
\hline Neurogenic tumor & $1(50 \%)$ & $1(50 \%)$ & \\
\hline Other & $1(50 \%)$ & $1(50 \%)$ & \\
\hline
\end{tabular}

Bold values indicate statistically significant parameter

Table 3 Clinical outcomes of endoscopic submucosal dissection for the study subjects $(n=35)$

\begin{tabular}{ll}
\hline Resection & $n(\%)$ \\
\hline R0 & $26(74.3 \%)$ \\
R1 & $6(17.1 \%)$ \\
R2 & $3(8.6 \%)$ \\
Procedure time (min; mean \pm SD) & $32.29 \pm 20.55$ \\
Major bleeding, $n(\%)$ & $0(0 \%)$ \\
Perforation, $n(\%)$ & $2(5.7 \%)$ \\
\hline
\end{tabular}

layer was a factor related to complete resection, whereas the tumor size and location were not. They performed EUS to assess the connection area between the tumor and the muscularis propria layer. In the present study, a positive rolling sign and tumor size $\leq 2 \mathrm{~cm}$ were found to be factors related to complete resection. A positive rolling sign indicates that the tumor originated from the submucosal layer or has a very narrow connection (or no connection) to the muscularis propria layer. In the present study, all tumors that had a positive rolling sign were SETs that originated from the muscularis propria layer and had a narrow muscular connection. When the hyperechoic line between the tumor and the muscularis propria layer was found to be $\geq 50 \%$ of the maximal diameter of the tumor base by EUS, a narrow muscular connection was confirmed. Because the hyperechoic line was either not observed or was found to be $<50 \%$ of the maximal diameter of the tumor base in some cases, it was not possible to consistently identify a narrow muscular connection by EUS alone. Białek et al. reported that EUS was only $73 \%$ accurate in determining the layer of origin of tumors. In the present study, in the cases of tumors that had a narrow muscular connection, a significant portion of the tumor base was loosely connected to the submucosal tissue (Fig. 3A, B). A higher R0 resection rate therefore was expected because of the relative ease of performing a dissection without tumor surface injury. In the cases of tumors that had a wide muscular connection, a substantial portion of the tumor base was tightly connected to the muscle layer (Fig. 3C). A greater risk of perforation or incomplete resection ( $\mathrm{R} 1$ or $\mathrm{R} 2$ ) therefore was expected because dissection is performed on a large area of the muscularis propria layer.

Białek et al. reported a $68 \% \mathrm{R} 0$ resection rate for tumors that originated from the muscularis propria layer, whereas $\mathrm{Li}$ et al. reported a $94 \% \mathrm{R} 0$ resection rate. The overall R0 resection rate in the present study was $74.3 \%$. The R0 resection rate for tumors with a positive rolling 
Table 4 Predictive parameters of perforation in patients who underwent endoscopic submucosal dissection for gastric subepithelial tumors

\begin{tabular}{|c|c|c|c|}
\hline \multirow[t]{2}{*}{ Parameters } & \multicolumn{2}{|l|}{ Perforation } & \multirow[t]{2}{*}{$p$ value } \\
\hline & Yes $(n=2)$ & No $(n=33)$ & \\
\hline Rolling sign, $n(\%)$ & & & 0.489 \\
\hline Positive & $0(0 \%)$ & $16(100 \%)$ & \\
\hline Negative & $2(10.5 \%)$ & $17(89.5 \%)$ & \\
\hline Mobility, $n(\%)$ & & & 0.017 \\
\hline Fixed lesion & $2(40.0 \%)$ & $3(60.0 \%)$ & \\
\hline Mobile lesion & $0(0 \%)$ & $30(100 \%)$ & \\
\hline Connection with fourth layer on EUS, $n(\%)$ & & & 0.536 \\
\hline Narrow connection & $0(0 \%)$ & $12(100 \%)$ & \\
\hline Wide connection & $2(8.7 \%)$ & $21(91.3 \%)$ & \\
\hline Tumor size, $n(\%)$ & & & 0.999 \\
\hline$\leq 20 \mathrm{~mm}$ & $1(4.3 \%)$ & $22(95.7 \%)$ & \\
\hline$>20 \mathrm{~mm}$ & $1(8.3 \%)$ & $11(91.7 \%)$ & \\
\hline Histological diagnosis, $n(\%)$ & & & 0.002 \\
\hline Leiomyoma & 0 & 21 & \\
\hline GIST & 0 & 10 & \\
\hline Neurogenic tumor & 2 & 0 & \\
\hline Other & 0 & 2 & \\
\hline
\end{tabular}

Bold values indicate statistically significant parameter

sign $(93.8 \%)$ was significantly higher than that for tumors with a negative rolling sign $(57.9 \%)$. Białek et al. reported that tumor size was not related to the complete resection rate. In the present study, the $\mathrm{R} 0$ resection rate for tumors $\leq 2 \mathrm{~cm}$ in size ( $85 \%$ ) was significantly higher than that for tumors $>2 \mathrm{~cm}$ in size $(50 \%)$. In theory, as the dissection area increases in proportion to the tumor size, the possibility of incomplete resection also increases. Further studies involving more cases are needed to test this hypothesis.

Complications related to treatment, both of which were instances of perforation, occurred in two cases. Fixed tumor mobility and the presence of a histologically diagnosed neurogenic tumor appeared to be factors related to the occurrence of perforation.

When a tumor was found to be fixed or immobile upon palpation with biopsy forceps, a broad muscular connection was shown by EUS. High tumor mobility is expected in the case of a submucosal growing tumor with a narrow muscular connection. Lower tumor mobility is expected in the case of a submucosal growing tumor with a broad muscular connection or in the case of an intramural-type or subserosal growing tumor (Fig. 4). A subserosal growing tumor shows an extraluminal bulging pattern on EUS and is easily diagnosed. In contrast, an intramural-type tumor is difficult to diagnose by EUS, and the accuracy of EUS diagnosis in terms of the degree of muscular connection is unsatisfactory. The measurement of tumor mobility and the detection of a positive rolling sign, in addition to EUS findings, therefore will be helpful in predicting the success rate of endoscopic resection or the occurrence of complications.

In the present study, perforation occurred in cases of schwannoma and neurilemoma, both of which are neurogenic tumors. This finding is consistent with findings reported in other studies [15]. In cases of gastric schwannoma, the intramural type has been reported to be the most common. It is difficult to dissect intramural-type schwannoma from the adjacent muscle tissue because, in contrast to soft tissue schwannoma, there is no encapsulation and perforation therefore occurs frequently $[16,17]$.

In cases of intermediate or small SETs that originate from the muscularis propria layer, it is difficult to choose an optimal treatment strategy because preoperative diagnosis and the assessment of the malignant potential of these tumors also are difficult. Endoscopic resection of SETs is less invasive than surgical resection and has high diagnostic accuracy. However, endoscopic resection is not widely used because of its disadvantages, particularly the high risk of incomplete resection and of complications, including perforation. The present study was conducted to analyze factors related to the complete resection rate of endoscopic resection and to the occurrence of complications and to investigate the appropriate indications and contraindications of endoscopic resection. We found that tumors $\leq 2 \mathrm{~cm}$ in size or with a positive rolling sign showed high complete resection rates and almost no complications. Such tumors are therefore presumed to be ideal candidates for endoscopic resection. Endoscopic 

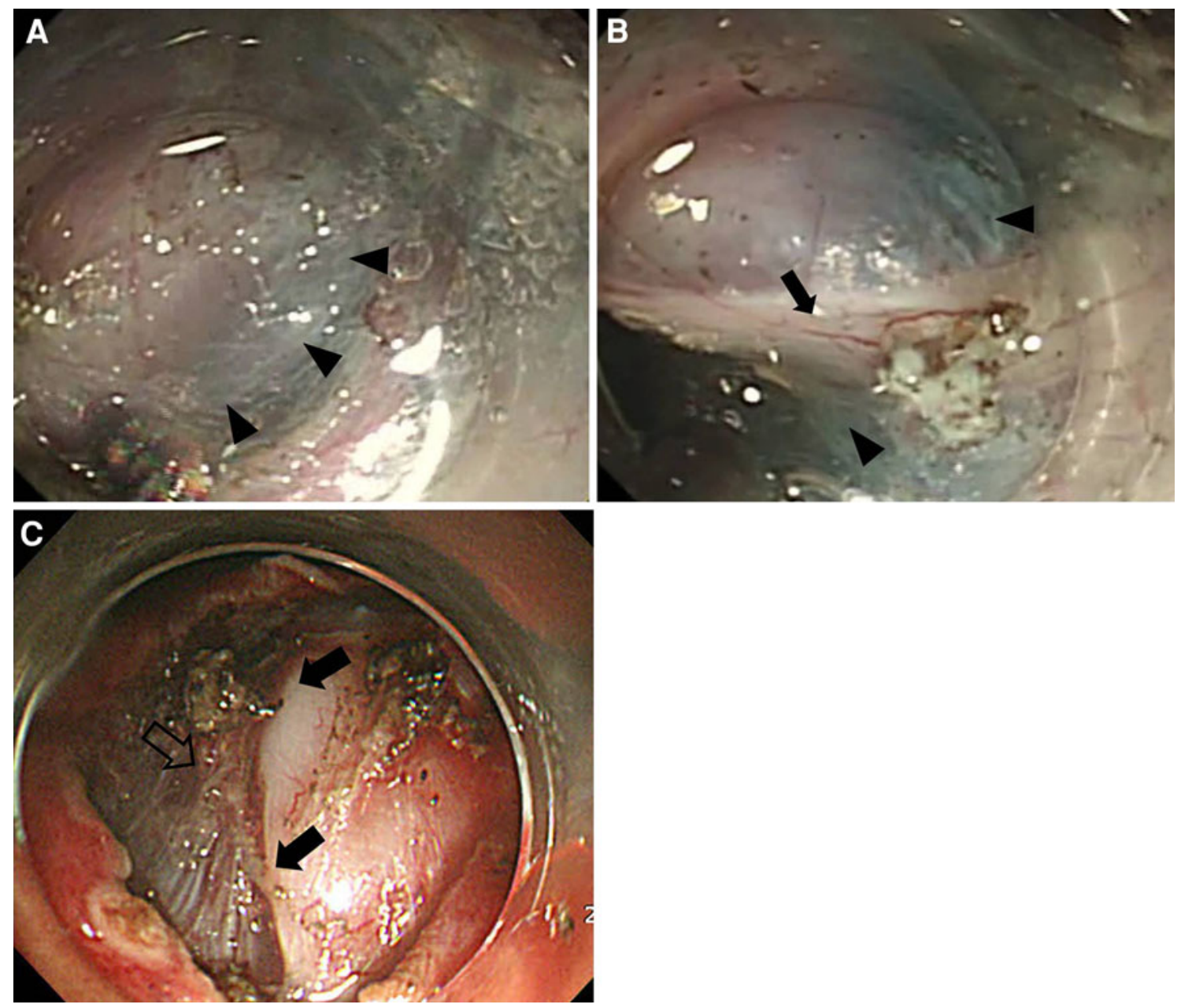

muscular connection area (black arrow) was observed. C Wide

Fig. 3 Endoscopic findings obtained from the endoscopic submucosal dissection of a gastric subepithelial tumor. A The tumor base was surrounded by a bluish submucosal layer (black arrowheads) following the injection of a mixture of glycerol and indigo carmine. B Following the dissection of the submucosal layer, a whitish narrow

muscular connection area (black arrows) and a muscularis propria layer (empty arrow) adjacent to the tumor were exposed following a circumferential incision around the lesion

Fig. 4 Schematic illustrations of subepithelial tumors with differing growing patterns and muscular connections.

A Subserosal. B Submucosal with a wide muscular connection. C Submucosal with a narrow muscular connection. D Intramural type
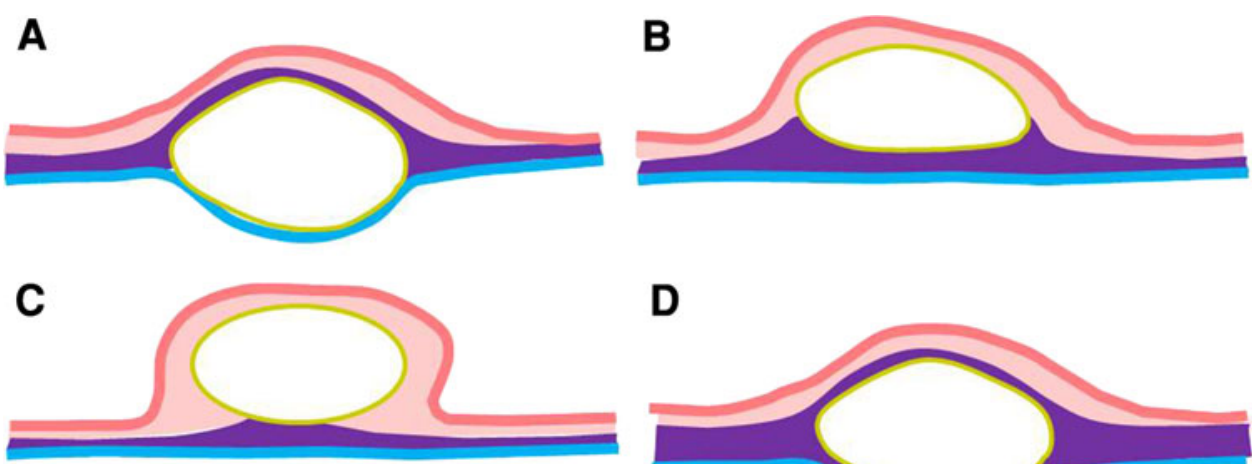

D

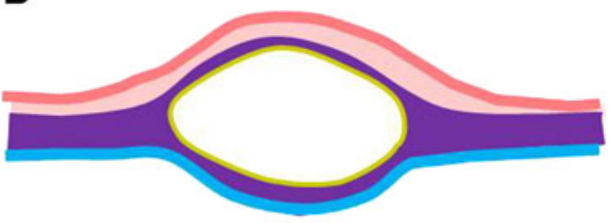

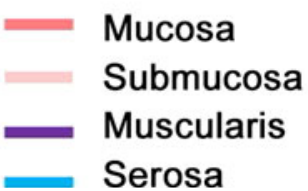


resection should not be considered in cases of neurogenic tumors or tumors that have no mobility.

The present study has some limitations. First, in view of the small number of patients studied, it is difficult to generalize our results. Although GIST is known as the most common SET, leiomyoma is more common than GIST in our results. Because endoscopically resected SETs were relatively small in size, we could not exclude the possibility of selection bias. Second, a long-term follow-up study has not yet been completed. In cases of GIST in particular, it is desirable to establish a management strategy following endoscopic resection based on the results of a long-term follow-up study. For the patients who underwent endoscopic resection, a regular follow-up study is underway, and the results of a long-term follow-up study involving the GIST patients in particular will be available later. The present study suggests that endoscopic resection (i.e., ESD) may be a good alternative to surgical resection for the treatment of SETs, including GISTs, that originate from the muscularis propria layer. Although this study provides a preliminary analysis of the appropriate indications for ESD, further studies are needed to confirm or extend our findings.

Disclosures S. Y. Chun, K. O. Kim, D. S. Park, I. J. Lee, J. W. Park, S. H. Moon, I. H. Baek, J. H. Kim, C. K. Park, and M. J. Kwon have no conflict of interest or financial ties to disclose.

Open Access This article is distributed under the terms of the Creative Commons Attribution License which permits any use, distribution, and reproduction in any medium, provided the original author(s) and the source are credited.

\section{References}

1. Hedenbro JL, Ekelund M, Wetterberg P (1991) Endoscopic diagnosis of submucosal gastric lesion: the results after routine endoscopy. Surg Endosc 5:20-23

2. Hwang JH, Rulyak SD, Kimmey MB (2006) American Gastroenterological Association Institute technical review on the management of gastric subepithelial masses. Gastroenterology 130:22 $17-2228$

3. Chak A (2002) EUS in submucosal tumors. Gastrointest Endosc $56: \mathrm{S} 43-\mathrm{S} 48$
4. Hwang JH, Kimmey MB (2004) The incidental upper gastrointestinal subepithelial mass. Gastroenterology 126:301-307

5. Kawamoto K, Yamada Y, Utsunomiya T, Okamura H, Mizuguchi M, Motooka M, Hirata N, Watanabe H, Sakai K, Kitaqawa S, Kinukawa N, Masuda K (1997) Gastrointestinal submucosal tumors: evaluation with endoscopic US. Radiology 205:733-740

6. Karaca C, Turner BG, Cizginer S, Forcione D, Brugge W (2010) Accuracy of EUS in the evaluation of small gastric subepithelial lesions. Gastrointest Endosc 71:722-727

7. Lee CK, Chung IK, Lee SH, Lee SH, Lee TH, Park SH, Kim HS, Kim SJ, Cho HD (2010) Endoscopic partial resection with the unroofing technique for reliable tissue diagnosis of upper GI subepithelial tumors originating from the muscularis propria on EUS (with video). Gastrointest Endosc 71:188-194

8. Kojima T, Takahashi H, Parra-Blanco A, Kohsen K, Fujita R (1999) Diagnosis of submucosal tumor of the upper GI tract by endoscopic resection. Gastrointest Endosc 50:516-522

9. Cantor MJ, Davila RE, Faigel DO (2006) Yield of tissue sampling for subepithelial lesions evaluated by EUS: a comparison between forceps biopsies and endoscopic submucosal resection. Gastrointest Endosc 64:29-34

10. Imagawa A, Okada H, Kawahara Y, Takenaka R, Kato J, Kawamoto H, Fujiki S, Takata R, Yoshino T, Shiratori Y (2006) Endoscopic submucosal dissection for early gastric cancer: results and degrees of technical difficulty as well as success. Endoscopy 38:987-990

11. Białek A, Wiechowska-Kozłowska A, Jan Pertkiewicz J, Polkowski M, Milkiewicz P, Karpińska K, Ławniczak M, Starzyńska $T$ (2012) Endoscopic submucosal dissection for treatment of gastric subepithelial tumors (with video). Gastrointest Endosc 75: 276-286

12. Park YS, Park SW, Kim TI, Song SY, Choi EH, Chung JB, Kang JK (2004) Endoscopic enucleation of upper-GI submucosal tumors by using an insulated-tip electrosurgical knife. Gastrointest Endosc 59:409-414

13. Lee IL, Lin PY, Tumg SY, Shen CH, Wei KL, Wu CS (2006) Endoscopic submucosal dissection for the treatment of intraluminal gastric subepithelial tumors originating from the muscularis propria layer. Endoscopy 38:1024-1028

14. Edge SB, Byrd DR, Compton CC, Fritz AG, Greene FL, Trotti A (eds) (2009) AJCC cancer staging manual, 7th edn. Springer, New York

15. Jeong ID, Jung SW, Bang SJ, Shin JW, Park NH, Kim do H (2011) Endoscopic enucleation for gastric subepithelial tumors originating in the muscularis propria layer. Surg Endosc 25(2): 468-474

16. Bruneton JN, Drouillard J, Roux P, Ettore F, Lecomte P (1983) Neurogenic tumors of the stomach. Report of 18 cases and review of the literature. Rofo 139:192-198

17. Voltaggio L, Murray R, Lasota J, Miettinen M (2012) Gastric schwannoma: a clinicopathologic study of 51 cases and critical review of the literature. Hum Pathol 43(5):650-659 